\title{
OPTICAL MEASUREMENTS OF WATER LENSES IN ICE
}

\author{
By M.E.R. WALFORD, D.W. ROBERTS, and I. HILL
}

(H.H. Wills Physics Laboratory, University of Bristol, Bristol BS8 1TL, England)

ABSTRACT. The dihedral angle of water at a grain boundary in ice is found, by measuring the optical focal length of lenticular water inclusions, to be $33.6 \pm 0.7^{\circ}$. The new result leads to only minor revision of published experimental values of specific surface free energies in the icewater system (Ketcham and Hobbs, 1969).

\section{INTRODUCTION}

After polycrystalline ice has been stressed at the melting point for a short time, groups of lenticular water inclusions can be found at certain of the grain boundaries (Nye and Mae, 1972). Once the stress is relaxed, there is a period of thermal annealing during which the larger lenses grow at the expense of the smaller ones; in well-annealed ice the lenses are typically $20-200 \mu \mathrm{m}$ in diameter. The equilibrium shape of a lens of given volume is determined by the balance of surface tensions acting at its rim (Fig. 1), and it follows that

$$
2 \gamma_{\mathrm{IW}} \cos \theta=\gamma_{\mathrm{GB}}
$$

where $\gamma_{I W}$ is the surface free energy of the ice-water interface, $\gamma_{\mathrm{GB}}$ is that of the grain boundary and $2 \theta$ is the dihedral angle measured in the water phase.

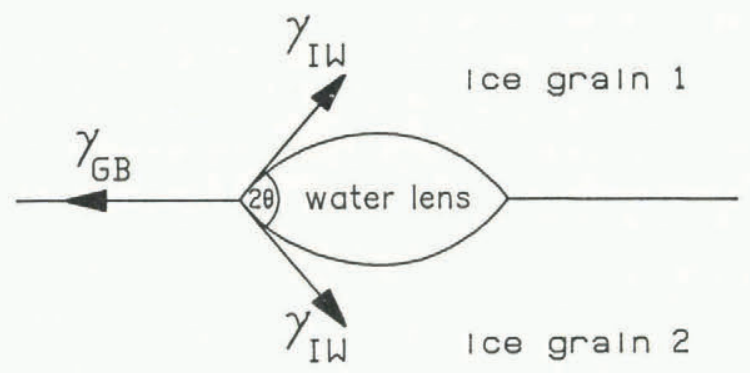

Fig 1. A water lens at a grain boundary in ice.

The refractive index of water is slightly greater than that of ice (we may neglect the slight birefringence of ice), so that a lenticular water inclusion behaves optically as a converging lens. As we shall discuss later, although the water lenses are geometrically thick, they behave optically as thin lenses, because $\left(\mu_{\mathrm{IW}}-1\right) \ll 1$, where $\mu_{\mathrm{IW}}$ is the refractive index for an ice-water interface. Accordingly we find

$$
4 f \theta\left(\mu_{\mathrm{IW}}-1\right)=d
$$

where $d$ is the lens diameter and $f$ is the focal length measured in ice. We can determine $\theta$ by measuring $d$ and $f$ with a travelling microscope as shown in Figure 2. Parallel

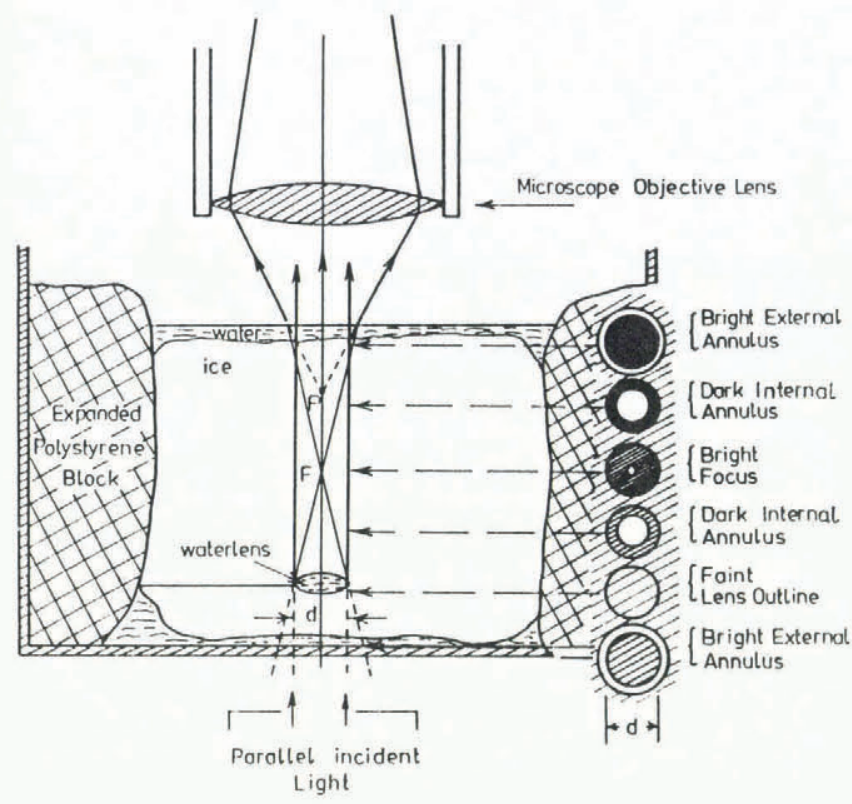

Fig. 2. Ray-optical diagram of refraction by water lenses in ice, illustrating the image seen in the microscope for different positions of its plane of focus.

light is incident from below on to the horizontal plane containing the water lenses. The microscope is focussed on the plane containing the water lens and a measurement is made of the lens diameter $d$. It is then raised until the focus of the water lens is found. The vertical distance travelled is equal to the apparent focal length of the water lens in ice and must be multiplied by the refractive index of ice in order to give $f$. The experiment was originally carried out by Walford, who found the value $2 \theta=32 \pm 3^{\circ}$, which was reported in Nye and Mae (1972). It has recently been repeated with some improvements by Hill and Roberts, who obtained the value $33.6 \pm 0.7^{\circ}$ (Hill, unpublished; Roberts, unpublished).

\section{EXPERIMENTAL DETAILS}

Suitable samples of polycrystalline ice are prepared by the slow freezing of distilled water, continuously stirred to reduce bubble formation. The resulting ice contains crystals a few millimetres across, elongated in the growth direction. Rectangular blocks approximately $2 \mathrm{~cm} \times 3 \mathrm{~cm} \times 5 \mathrm{~cm}$ are cut, polished by melting against a warm metal plate, stressed in uniaxial compression at approximately 1 bar for $10 \mathrm{~s}$, and left to anneal in iced water for $1 \mathrm{~h}$. Then a chosen ice sample, orientated such that a suitable field of 
water lenses is horizontal, is jammed in a Perspex box by means of blocks of expanded polystyrene. It is covered to a depth of $2 \mathrm{~cm}$ with iced water, to reduce the unwanted distorting effects of refraction at the uneven surfaces of the block.

The lenses themselves are most clearly seen in white light, collimated to approximately $10^{-2}$ rad. Figure 3 shows the appearance of the lenses when the microscope is focussed (a) slightly below, and (b) slightly above the grain boundary. Lenses exactly in focus are almost invisible. It will be noticed that with the microscope focussed below the lens field, each lens appears slightly darker than the background and surrounded by a bright ring (the "Becke line") of internal diameter $d$. When the microscope is focussed above the lens field, the lens image consists of a bright disc surrounded by a dark ring of external diameter $d$.
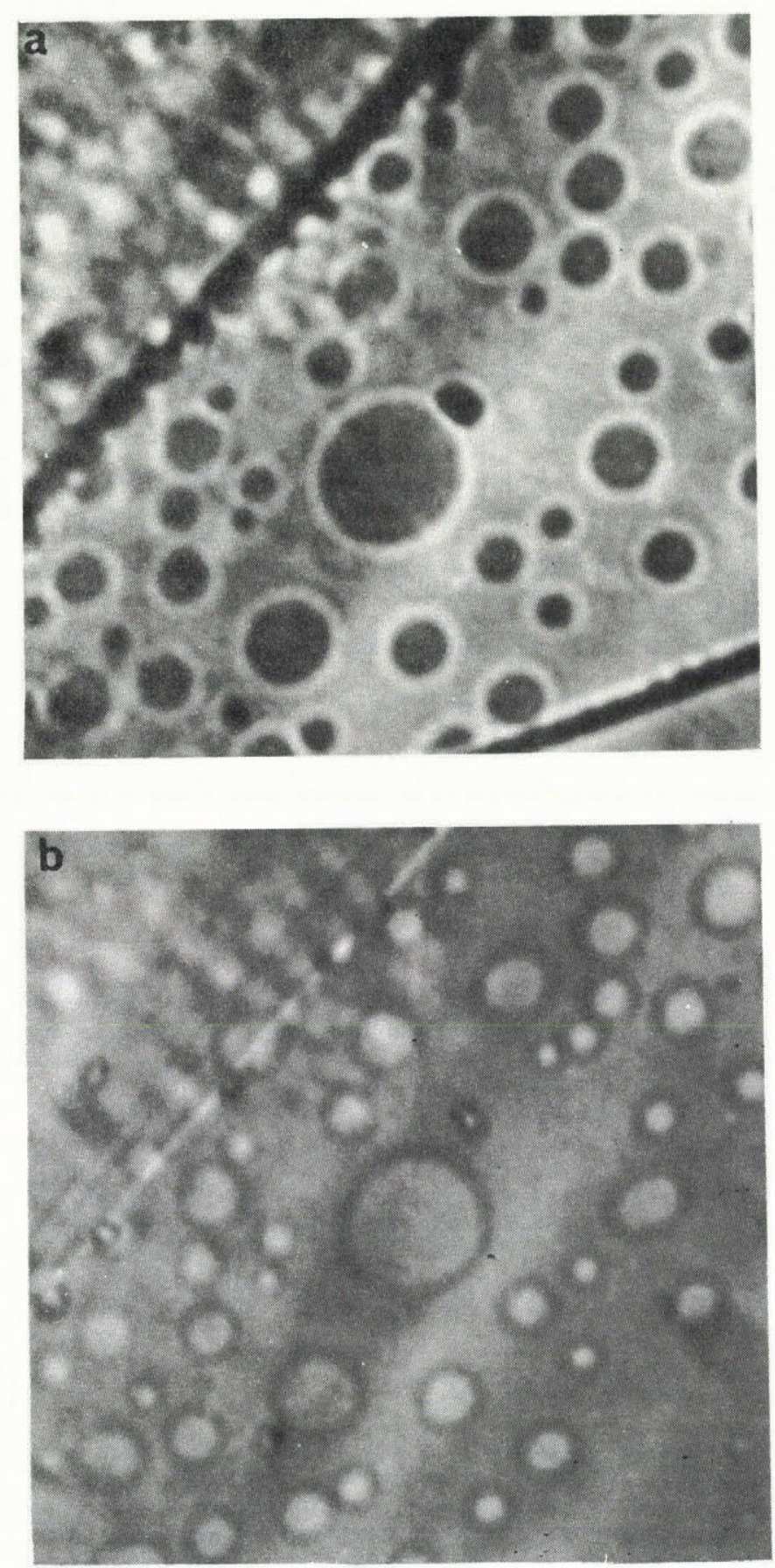

Fig. 3. Water-lens images photographed in white, paraxial illumination with the plane of focus of the microscope (a) slightly below, and (b) slightly above the lens plane. The diagonal lines are produced by a nearby water vein.
According to thin-lens ray-optical theory, the thickness of the annular Becke line, $b$, is given by

$$
b=2 \theta\left(\mu_{\mathrm{IW}}-1\right) \mu_{\mathrm{IA}} h
$$

where $h$ is the apparent height above the grain boundary at which the microscope is focussed. By measuring $b$ as a function of $h$, we obtain a preliminary value for $2 \theta$ of $33 \pm 3^{\circ}$

A better value can be obtained by measuring the diameter and then the apparent focal length of the water lens. The diameter is easily measured from photographs taken in white light but it is more difficult to find the height at which an image produced by a water lens is focussed, because the effects of diffraction are relatively large for such small lenses. For this purpose, a helium-neon laser is a suitable source of illumination. Unwanted modes are removed by focussing the laser light through a bloomed microscope objective lens on to a spatial filter carrying a $100 \mu \mathrm{m}$ diameter pin-hole. The well-defined focus of the laser beam acts as an object placed $60 \mathrm{~cm}$ in air below the sample. The apparent height of the conjugate image formed by the water lens is found from a sequence of photographs taken with the microscope at different closely separated heights. Even in the presence of diffraction, the required image position is where the disc of illumination produced by the convergent water lens is smallest (Born and Wolf, 1970 , p. 440). (This is true, because spherical aberration can be shown to be negligible in these experiments; in spite of the large dihedral angle of the presumably spherically curved water lenses, the small refractive index contrast makes the focal length for marginal rays differ by only $4 \%$ from the focal length for central rays.) A value for the dihedral angle of $33.6 \pm 0.7^{\circ}$ was obtained on carrying out measurements on 14 different lenses in two different fields, the lens diameters ranging from 65 to $115 \mu \mathrm{m}$.

An interesting, unexpected observation (of marginal statistical significance) is that the dihedral angle appears to increase systematically by a few degrees as the lens diameter increases from 65 to $115 \mu \mathrm{m}$. The effect may arise because of the slow, continuous annealing of a field of lenses of different sizes. However, no changes in lens size or focal length were directly observed during the course of the measurements. The effect is too small to constitute a severe source of systematic error.

Attempts to improve the precision of measurement of the focus by examining the detailed structure of the diffraction pattern in the vicinity of the focus were frustrated by what appeared to be radiation diffracted around the outside of the lens.

No account was taken in the present work of any possible effects of the crystallographic orientation of the two ice crystals on the lens shapes, but the absence of observable astigmatism in a horizontal lens implies that such effects are small. We hope this may form the subject of further research.

\section{DISCUSSION}

The measurement of $\theta$ has significance in connection with the experimental measurement of interfacial free energies in the ice-water-vapour system. Knowing $\gamma_{W V}$, the surface free energy of water, Ketcham and Hobbs (1969) made three independent measurements of suitable interfacial angles and so obtained values for $\gamma_{I V}, \gamma_{I W}$, and $\gamma_{\text {GB }}$, the specific interfacial free energies associated with the ice-vapour, the ice-water, and the grain-boundary interfaces, respectively. The present measurement of $2 \theta$ is probably to be preferred to the corresponding measurement by Ketcham and Hobbs (1969) of $20 \pm 10^{\circ}$, which was based on observations of dihedral angle where an intergranular vein of water crops out on the surface of polycrystalline ice. The angle so measured is probably not the same as that which would be measured at depth. The effect of using the present value of $33.6 \pm 0.7^{\circ}$ is to cause a small upward revision from 33 to $34 \mathrm{~mJ} \mathrm{~m}^{-2}$ in the value of $y_{\text {IW }}$. The precision of the result remains limited by the difficulty of measuring the angle of contact of water on ice in the presence of vapour. Hobbs (1974) gave a useful discussion of the problem. 


\section{ACKNOWLEDGEMENT}

We wish to acknowledge that the inspiration for this work was provided originally by Professor J.F. Nye. A helpful observation was made by Dr V.J. Smith.

\section{REFERENCES}

Born, M., and Wolf, E. 1970. Principles of optics. Fourth edition. Oxford, Pergamon Press.
Hill, I. Unpublished. Investigation into the surface energies of water substance through investigation of intergranular water lenses. [B.Sc. thesis, University of Bristol, 1986.]

Hobbs, P.V. 1974. Ice physics. Oxford, Clarendon Press.

Ketcham, W.M., and Hobbs, P.V. 1969. An experimental determination of the surface energies of ice. Philosophical Magazine, Eighth Ser., Vol. 19, No. 162, p. 1161-73.

Nye, J.F., and Mae, S. 1972. The effect of non-hydrostatic stress on intergranular water veins and lenses in ice. Journal of Glaciology, Vol. 11, No. 61, p. 81-101.

Roberts, D.W. Unpublished. Investigation of surface free energies in ice water systems. [B.Sc. thesis, University of Bristol, 1986.]

MS. received 26 November 1986 